\title{
New Results of Moscow Cepheid Radial Velocity Programme
}

\author{
N. A. Gorynya ${ }^{1}$, N. N. Samus ${ }^{1}$, M. E. Sachkov ${ }^{1}$, S. V. Antipin ${ }^{2}$, \\ A. S. Rastorgouev ${ }^{2}$
}

\begin{abstract}
A sequence similar to Hertzsprung progression was revealed for Cepheid radial-velocity curves. We separated two pulsation modes for six double-mode Cepheids and determined radii for five of them. Several new spectroscopic-binary Cepheids were discovered; we present new preliminary orbital periods for V496 Aql, VY Cyg, and V1334 Cyg, in a combined table of our results on Cepheid binarity.
\end{abstract}

Since 1987, we have been carrying out a large program of measuring radial velocities (RVs) of Cepheids using a CORAVEL-type spectrometer. By now, we have measured about $6760 \mathrm{RVs}$ for more than 140 Cepheids. Three catalogues have been published (Gorynya et al. 1998 and references therein). The revised combined data of the three catalogues will be soon made available via INTERNET; the third catalogue can already be retrieved from:

http://www.sai.msu.su/groups/cluster/gcvs/gcvs/cep/

where the combined catalogue will also be deposited. These data, one of the richest bodies of original accurate RVs for Cepheids, can be used for studies of morphology of RV curves, for Baade-Wesselink studies, for research on the Galaxy's kinematics and dynamics, and for discoveries of spectroscopic binaries.

Recently, a Hertzsprung sequence of RV curves for classical Cepheids has been derived (Gorynya 1998). The similar sequence for light curves is wellknown; but RV curves are easier to interpret theoretically, so this form of the sequence deserves special attention.

Thanks to the good coverage of the curves, it has become possible to separate pulsation modes for double-mode Cepheids based on RV curves only. We have separated modes for EW Sct, CO Aur, TU Cas, V367 Sct, BQ Ser, and BD $-10^{\circ} 4669$ (Samus \& Gorynya 1991, Sachkov 1997, Antipin et al. 1999). Using the modification of Balona's method described in Sachkov (1997), we derived the radius of $\mathrm{BD}-10^{\circ} 4669: \log R \approx 1.76$, in agreement with the value 1.73 predicted from the period-radius relation derived by Sachkov (1997). In the cited paper, Sachkov presents radii for CO Aur, TU Cas, EW Sct, and BQ Ser.

Our original Cepheid RVs are very effective for discoveries of new spectroscopic binaries (SBs), and for confirmations of suspected SBs. We have discovered, or definitely confirmed, spectroscopic binarity for $\mathrm{MW} \mathrm{Cyg,} \mathrm{VZ} \mathrm{Cyg,} \mathrm{and}$ BY Cas, and have published orbital solutions for a number of stars (Gorynya et al. 1996; Rastorgouev et al. 1997). Our new SB discovery is VY Cyg. Our main results on SB Cepheids are summarised in Table 1.

\footnotetext{
${ }^{1}$ Institute of Astronomy, 48 Pyatnitskaya Str., Moscow 109017, Russia

${ }^{2}$ Sternberg Astronomical Institute, 13 Universitetsky Ave., Moscow 119899, Russia
} 
This work was supported, in part, by the Russian Foundation for Basic Research and by the Federal Programme "Astronomy".

Table 1. Spectroscopic Binary Cepheids

\begin{tabular}{lllllll}
\hline Cepheid & $\begin{array}{l}P_{\text {puls }} \\
\text { days }\end{array}$ & $\begin{array}{l}P_{\text {orb }} \\
\text { days }\end{array}$ & $\begin{array}{l}a \sin i \\
\text { au }\end{array}$ & $\begin{array}{l}f(m) \\
M_{\odot}\end{array}$ & $\begin{array}{l}M_{1} \\
M_{\odot}\end{array}$ & $M_{\odot}$ \\
\hline FF Aql & 4.4709 & 1434.2 & 0.67 & 0.02 & 5.5 & $\geq 1 \pm 0.2$ \\
V496 Aql & 6.8072 & $1447(573)$ & & & & \\
RX Cam & 7.9122 & 1116 & 1.2 & 0.19 & 6.5 & $\geq 2.5 \pm 0.2$ \\
SU Cas & 1.9495 & 407.2 & 0.13 & 0.002 & 4 & \\
BY Cas & 3.2223 & 563 & & 0.04 & & \\
DL Cas & 8.0007 & 684.4 & & 0.2784 & & \\
SU Cyg & 3.8456 & 549.25 & 1.42 & 1.27 & 5.0 & $\geq 5$ \\
VY Cyg & 7.8570 & 941 & & & & \\
VZ Cyg & 4.8644 & 1483 & 1.39 & 0.16 & 5.5 & $\geq 2 \pm 1.5$ \\
MW Cyg & 5.9547 & 437.3 & 0.25 & 0.011 & 6 & $\geq 0.8 \pm 0.05$ \\
V532 Cyg & 3.2838 & $388 ?$ & & $0.0001 ?$ & & \\
V1334 Cyg & 3.3325 & $1947(1463)$ & & & & $\geq 1.25 \pm 0.15$ \\
TX Del & 6.1659 & 133.3 & 0.17 & 0.037 & 6 & $\geq 0.8 \pm 0.1$ \\
Z Lac & 10.8860 & 376.9 & 0.54 & 0.14 & 6 & $\geq 2.7 \pm 0.2$ \\
T Mon & 27.0333 & 25000 & & & & \\
AU Peg & 2.4115 & 53.34 & 0.22 & 0.49 & 4 & $\geq 2.7 \pm 0.016$ \\
AW Per & 6.4636 & $1911 ?$ & & $0.016 ?$ & & \\
S Sge & 8.3823 & 675.75 & 0.93 & 0.23 & 6.5 & $\geq 1.1 \pm 0.05$ \\
V350 Sgr & 5.1539 & 1481.8 & 1.34 & 0.15 & 5.5 & $\geq 2.1 \pm 0.2$ \\
BQ Ser & 4.2707 & $136(1009)$ & & 0.005 & & \\
SZ Tau & 3.1489 & $1244 ?, 340 ?$ & & & & \\
EU Tau & 2.1025 & $980 ?$ & & & & \\
\hline
\end{tabular}

Remarks: $S U$ Cyg: A triple system. $T X$ Del: If a Pop. II Cepheid: $M_{1} \approx 0.7 M_{\odot} ; M_{2} \geq 0.35 \pm 0.05 M_{\odot}$. AU Peg: If a Pop. II Cepheid: $M_{1} \approx 0.7 M_{\odot} ;$ $M_{2} \geq 1.2 \pm 0.1 M_{\odot}$. AW Per: SB2. BQ Ser: $\mathrm{CEP}(\mathrm{B}), P_{0}=4.27073$, $P_{1}=3$ d. 012 .

\section{References}

Antipin, S. V., Gorynya, N. A., Sachkov, M. E., et al. 1999, IBVS, No. 4718 Gorynya, N. A. 1998, IBVS, No. 4636

Gorynya, N. A., Rastorgouev, A. S., \& Samus, N. N. 1996, Astron. Lett., 22, 33 Gorynya, N. A., Samus, N. N., Sachkov, M. E., et al. 1998, Astron. Lett., 24, 815

Rastorgouev, A. S., Gorynya, N. A., \& Samus, N. N. 1997, in Binary Stellar Systems, ed. A. G. Massevich (Moscow: Kosmosinform), 123

Sachkov, M. E. 1997, IBVS, No. 4484

Samus, N. N. \& Gorynya, N. A. 1991, Astron. Tsirk., No. 1550, 25 